\title{
Effect of nanoclay on improved rheology properties of polyacrylamide solutions used in enhanced oil recovery
}

\author{
Goshtasp Cheraghian · Seyyed Shahram Khalili Nezhad • \\ Mosayyeb Kamari • Mahmood Hemmati • \\ Mohsen Masihi · Saeed Bazgir
}

Received: 28 February 2014/ Accepted: 10 June 2014/Published online: 3 July 2014

(C) The Author(s) 2014. This article is published with open access at Springerlink.com

\begin{abstract}
Recently, a renewed interest arises in the application of nanotechnology for the upstream petroleum industry. In particular, adding nanoparticles to fluids may drastically benefit enhanced oil recovery (EOR) and improve well drilling, by changing the properties of the fluid, rocks wettability alteration, advanced drag reduction, strengthening the sand consolidation, reducing the interfacial tension and increasing the mobility of the capillary trapped oil. In this study, we focus on roles of clay nanoparticles on polymer viscosity. Polymer-flooding schemes for recovering residual oil have been in general less than satisfactory due to loss of chemical components by adsorption on reservoir rocks, precipitation, and resultant changes in rheological properties. Rheological properties' changes are mainly determined by the chemical structure and mix of the polymers, surface properties of the rock, composition of the oil and reservoir fluids, nature of the added polymers and solution conditions such as salinity,
\end{abstract}

G. Cheraghian $(\bowtie) \cdot$ S. S. Khalili Nezhad

Department of Petroleum and Gas Engineering, Science and Research Branch, Islamic Azad University, Tehran, Iran

e-mail: goshtasbc@gmail.com

M. Kamari

Chemical Engineering Department, Sahand University

of Technology, Tabriz, Iran

M. Hemmati

Research Institute of Petroleum Industry (RIPI), Tehran, Iran

M. Masihi

Chemical and Petroleum Engineering Department, Sharif

University of Technology, Tehran, Iran

S. Bazgir

Department of Polymer Engineering, Science and Research Branch, Islamic Azad University, Tehran, Iran
$\mathrm{pH}$ and temperature. On the other hand, in this study, the focus is on viscosity and salinity of solutions polyacrylamide polymer solutions with different nanoparticles degrees and molecular weight. Results in certain range of clay concentration used in this test, the way of clay adding, have positive effects on solution viscosity. The effect of the polymer content and salinity were also to be investigated.

Keywords Enhanced oil recovery $\cdot$ Rheology $\cdot$ Nanoclay · Polymer viscosity $\cdot$ Polyacrylamide

\section{Introduction}

Polymer flooding is one of the most successful methods in enhanced oil recovery. The polymers that are mainly used in oilfields are water soluble polyacrylamide (PAM), xanthan gum (Xc), and associative polymer (AP). Polymer solutions, in contrast to water, exhibit non-Newtonian rheological behaviors, such as shear thinning and shear thickening effects, which lead to different viscosity properties in a reservoir, as compared with those in water flooding. When a polymer solution is injected into a reservoir from an injection well, the flow velocity, which is related to shear rate, will change from wellbore to in-depth of a reservoir; therefore, the polymer solution viscosity will also change from near wellbore to in-depth of a reservoir correspondingly (Sun et al. 2012). The volume of the polymer solution injected may be $50 \% \mathrm{PV}$, depending on the process design (Green and Willhite 1998; Satter et al. 2008). High molecular weight (HMW) polyacrylamide and its derivatives are widely used in oilfield applications ranging from drilling fluids, enhanced oil recovery, and treatment of oil sand tailings. In these applications, the adsorption characteristics of these 
polymers are essential since it would affect their applicability and efficiency (Al-Hashmi et al. 2013). Pancharoen (2009) studied on the physical properties of polymers used in enhanced oil recovery. The impact of increased salinity in the rheological behavior of polymer solution was examined. It was shown that by increasing the salinity of polymer solution, some properties such as polymer viscosity, inaccessible pore volume and permeability reduction factor are reduced. These studies were performed using associative polymers and it was shown that these polymers' behavior is similar to non-Newtonian fluids, and changes in their viscosity versus shear rate are a power law function of the fluid viscosity, and also they show good resistance to adverse effect of salinity. Finally, associative polymers were compared with conventional polymers. Magbagbeola (2008) validated the Universal Viscosity Model (UVM) for apparent viscosity of flowing polymer solution through the porous medium, and changes in the model parameters for the different polymers were studied and calculated. In that study, a partial hydrolyzed polyacrylamide and co-polymers were used. It was concluded that UVM fits the core flooding data in wide range of condition for polymer used in EOR. In that case, using the rheological data of polymer solutions and without the need for costly experiments the operating condition of the process can be analyzed. It was shown that some parameters such as permeability, polymer concentration, polymer molecular weight, and salinity affect the rheological properties of polymers through the porous medium. Delshad et al. (2008) developed and proposed a unified apparent viscosity model for the entire range of flow velocity. One major advantage of this model is that the dependence of polymer viscosity on the flow velocity through the porous medium can be estimated using only the bulk rheological properties of the polymer and petrophysical features of the porous media. Parameters that impact on these polymers include: shear rate, polymer concentration, sulfonate degree, molecular weight, $\mathrm{NaCl}$ concentration, divalent ion concentrations, and temperature. Finally, the results showed that these polymers can be considered as a substitute for hydrolyzed Polyacrylamide solution in high salinity and temperature (Rashidi 2009). Moradi (2011) performed the rheological measurements of two types of hydrolyzed polyacrylamides with different salinities. This polymer solution showed both the Newtonian behavior and shear thinning behavior. It was shown that salinity has a major impact on these polymers. The effect of polymer molecular weight and concentration of the polymer solution were analyzed, and the increase in viscosity due to these parameters was reported. Ghannam and Esmail (2002) studied the flowing behavior of alcoflood polymer solution, and their changes in viscosity and shear stress versus shear rate has been investigated. They showed that the apparent viscosity of the solution is a function of polymer concentration and shear rate. Their results also showed that the effect of polymer concentration at low shear rates is more sensible. With the passing of the era of easy oil and the increasing difficulty of finding new resources, attention of the traditional oil and gas industry has been directed to extract more resources from existing oil fields (enhanced oil recovery) and from the fields exposed to extremely harsh environments by using new technologies and solutions. Nanotechnology bears the promise and has received great attention recently from the petroleum industry and there is a general perception that more than a decade's nanotechnology hype may become nano reality in the oil field (Cheraghian et al. 2013).

Due to their characteristics, produced nanoparticles based on layered silicates such as montmorillonite are very important. Montmorillonite is comprised of many overlapping layers of crystal particles that generally have an average thickness of $0.96 \mathrm{~nm}$ and a diameter of 100-200 $\mathrm{nm}$. In this study, we develop a nano polymer formulation for optimum viscous at 25 and $80{ }^{\circ} \mathrm{C}$. In our work, we investigated using this nano clay to reduce the negative impacts caused by salinity on polyacrylamide fluids in EOR process.

\section{Materials and methodology}

\section{Materials}

Brine was prepared from reagent grade $\mathrm{NaCl}, \mathrm{MgCl}_{2}$, $\mathrm{Na}_{2} \mathrm{SO}_{4}$ and $\mathrm{CaCl}_{2}$ at concentrations ranging from 20,000 to $200,000 \mathrm{ppm}$.

All the polymers that used were commercially available, completely water soluble and differed mainly in their concentration (Table 1). In particular, we tested polyacrylamides that were similar in chemical structure and molecular weight of 14 million.

Local sodium bentonite with a mesh size of 200 and a particles size less than $75 \mu \mathrm{m}$ was used in this study. The

Table 1 Properties of examined samples

\begin{tabular}{llllr}
\hline $\begin{array}{l}\text { No. } \\
\text { ID }\end{array}$ & $\begin{array}{l}\text { Polymer content } \\
(\mathrm{ppm})\end{array}$ & $\begin{array}{l}\text { Clay content } \\
(\mathrm{wt} \%)\end{array}$ & \multicolumn{1}{c}{$\begin{array}{l}\text { Salinity } \\
(\mathrm{ppm})\end{array}$} \\
\hline 1 & B & 3,150 & 0 & 20,000 \\
2 & C & 225 & 0 & 20,000 \\
3 & D & 3,150 & 0 & 200,000 \\
4 & F & 3,150 & 0.9 & 200,000 \\
5 & G & 3,150 & 0.45 & 20,000 \\
6 & H & 3,150 & 0.45 & 200,000 \\
7 & K & 3,150 & 0.9 & 20,000 \\
\hline
\end{tabular}


Table 2 Chemical composition of bentonite

\begin{tabular}{lc}
\hline Formula & Wt \\
\hline $\mathrm{L} . \mathrm{O} .1$ & 13.2 \\
$\mathrm{Na}_{2} \mathrm{O}$ & 2.04 \\
$\mathrm{MgO}$ & 2.22 \\
$\mathrm{Al}_{2} \mathrm{O}_{3}$ & 14.59 \\
$\mathrm{SiO}_{2}$ & 61.03 \\
$\mathrm{SO}_{2}$ & 0.37 \\
$\mathrm{Cl}$ & 0.46 \\
$\mathrm{~K}_{2} \mathrm{O}$ & 0.76 \\
$\mathrm{CaO}$ & 0.77 \\
$\mathrm{TiO}_{2}$ & 0.22 \\
$\mathrm{Fe}_{2} \mathrm{O}_{3}$ & 2.09 \\
$\mathrm{BaO}$ & 0.11 \\
\hline
\end{tabular}

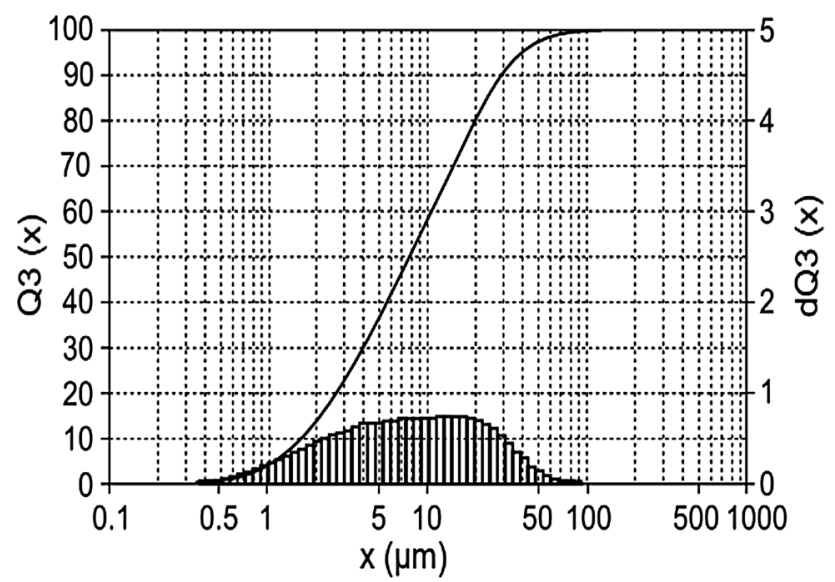

Fig. 1 Diagram of accumulative distribution of clay particles in feed before purification [6]

bentonite was produced in Iran and amended with the chemicals shown in Table 1. Oxford-ED2000 XRF and GC-2550TG were used for all chemical analyses (Table 2).

After the preparation and analysis of the raw materials, the clay was purified using a 2-inch hydrocyclone apparatus. To accomplish this, a suspension of $3 \mathrm{wt} \%$ clay in distilled water was prepared and then passed through the cyclone at a pressure of $0.15 \mathrm{MPa}$. This resulted in the removal of impure and large particles. Montmorillonite particles with a diameter of less than $6 \mu \mathrm{m}$ were then dried and used in the compatibility process. To ensure compatibility following the dispersion of clay in distilled water, a clay suspension was prepared and amended with 10, 20, 30, 40 or $50 \mathrm{wt} \%$ of the modifier materials "silane". The mixtures were then heated at $80{ }^{\circ} \mathrm{C}$ for $6 \mathrm{~h}$, after which the products were washed with distilled water and dried.

The distribution of clay particle sizes was measured before and after purification using a Laser Particle Size Analyzer (Figs. 1, 2). In addition, clay particles in the

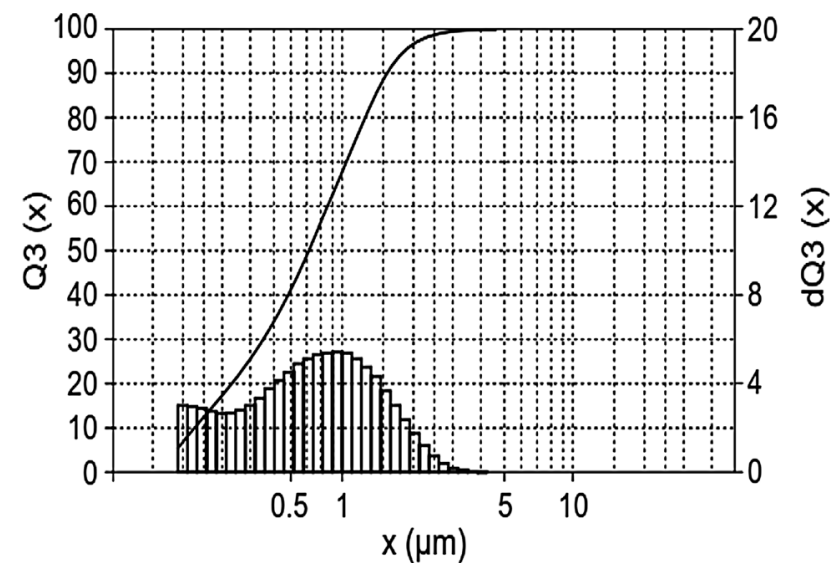

Fig. 2 Diagram of accumulative distribution of clay particles in feed after purification [6]

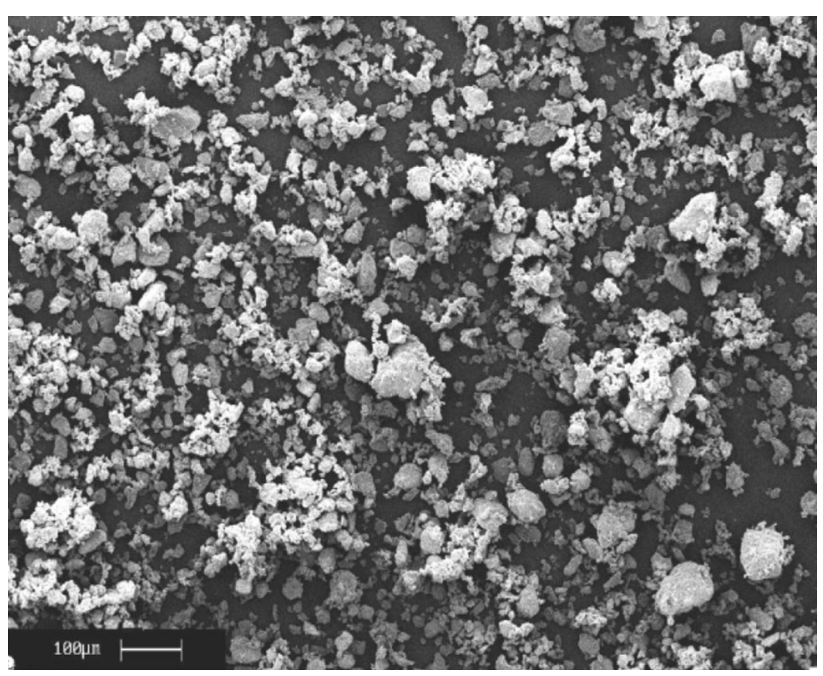

Fig. 3 SEM image of clay particles in feed before purification [6]

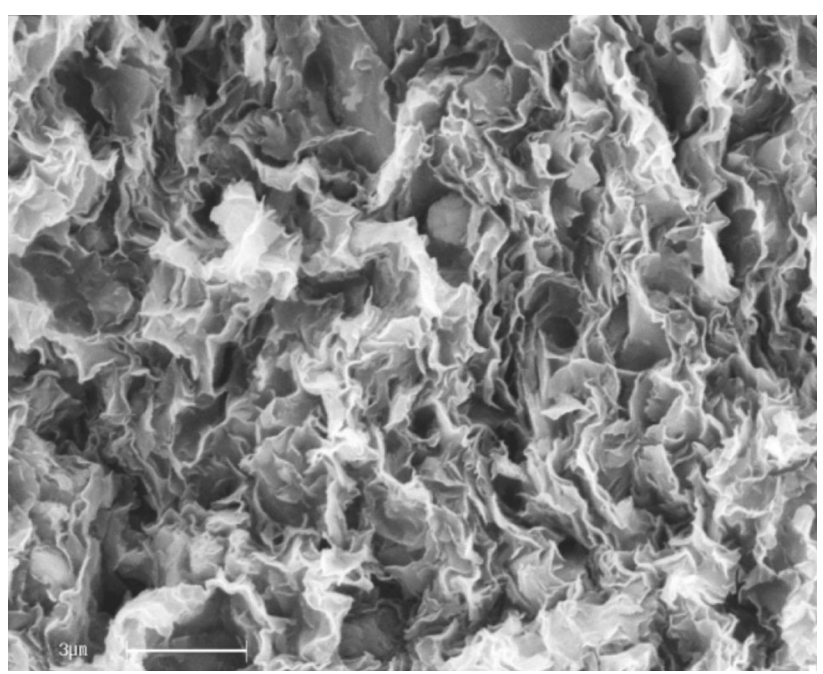

Fig. 4 SEM image of pure clay in magnetite [6] 
Fig. 5 XRD pattern, local pure sample [6]

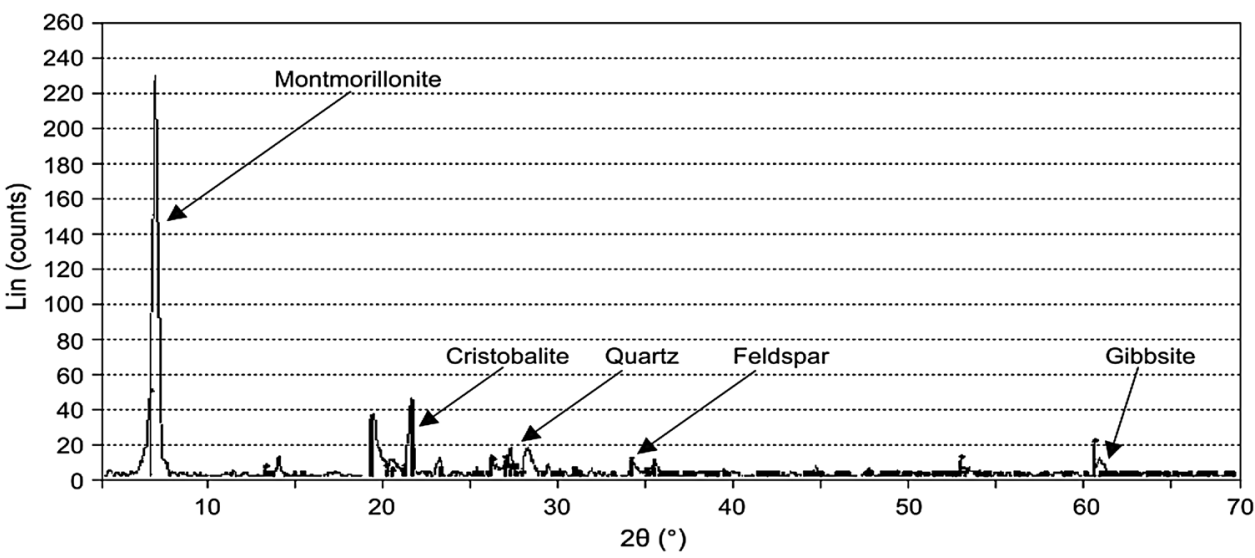

colloid state were analyzed by SEM (Figs. 3, 4). The intercalation of the samples was evaluated using an X-ray diffraction (Bazgir 2006).

Most of the clay particles are in the agglomerated form prior to purification. In addition, all of the clay particles had diameters of less than $75 \mu \mathrm{m}$. Furthermore, $50 \%$ of the particles in the clay were less than $7 \mu \mathrm{m}$ and $80 \%$ were less than $20 \mu \mathrm{m}$ in diameter.

The results of the XRD analysis revealed that most of the impurities, which included quartz, cristobalite, calcite, gibbsite and feldspar, were removed from the clay by the purification process. In addition, analysis of the particle size distribution revealed that most of the particles were less than $5 \mu \mathrm{m}$ in diameter and that greater than $80 \%$ of the particles were less than $2 \mu \mathrm{m}$ in diameter after the purification. Finally, the thickness of the layers in the crystal structure of montmorillonite and the patterns generated by XRD indicate that the clay was comprised of crystal layers on top of each other following purification (Fig. 5) (Khoeini et al. 2009).

\section{Methodology}

The most important property of a polymer is its ability to increase the solution's viscosity. The polymer solution exhibits non-Newtonian behavior, and its viscosity is a function of the shear rate. The viscosity of these polymer solutions was measured using a DV-III Ultra + Brookfield rheometer. The principle of operation of the DV-III Ultra is to drive a spindle (which is immersed in the test fluid) through a calibrated spring. The viscous drag of the fluid against the spindle is measured by the spring deflection. Spring deflection is measured with a rotary transducer. The viscosity measurement range of the DV-III Ultra (in centipoise) is determined by the rotational speed of the spindle, the size and shape of the spindle, the container the spindle is rotating in, and the full-scale torque of the calibrated spring. In each measurement, the shear rate was changed, and the effect of this change on the viscosity was measured.

\section{Results and discussion}

The effect of the polymer content

After that the two samples were prepared the effects of polymer concentration increment in low saline system were investigated. It is seen from the graph that increase in polymer concentration has positive impacts on solution and leads to viscosity increasing. In this case, simply one should express that having very low concentration of polymer, sample $\mathrm{C}$ variation graph for its viscosity regarding shear rate is different so that in high levels of this rate an increase in shear viscosity leads to solution increase (Fig. 6).

The effect of the solution salinity

To study salinity increment effects on polymer solution, two similar samples with different salinities were provided. Figure 7 shows that the negative effect of the salinity increasing on the system is so clear. Also, when the shear rate is low, this effect is the more obvious, and therefore an increase in the shear rate causes decreasing differences between two systems.

The effect of the salinity and clay concentration

Considering the previous test results, and clay presence effects on polymer solution and in case that to increase salinity imposes negative impacts on polymer solution, the following two samples were provided so that the system salinity is ten folded while having polymer and clay concentration in a fixed rate. Salinity level for the sample $G$ is 0.1 salinity of sample $\mathrm{H}$. It is seen that, by increasing the 
Fig. 6 Solution viscosity vs. shear rate, to evaluate the polymer concentration increment in low salinity system

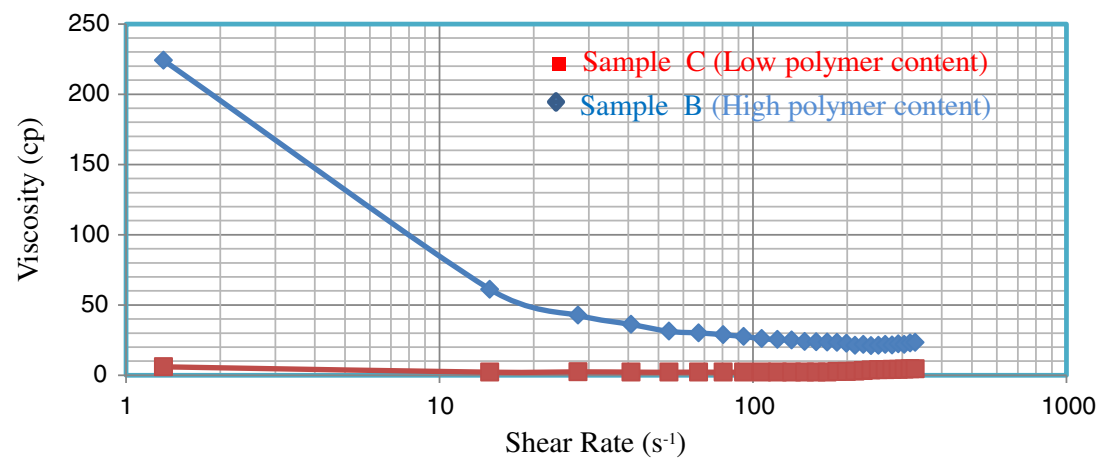

Fig. 7 Solution viscosity vs. shear rate, to evaluate the salinity effect

Fig. 8 Solution viscosity vs. shear rate, to evaluate effect of increasing salinity with constant polymer concentration and clay content


salinity level viscosity goes down (same as system without clay content) (Fig. 8).

The effect of the clay concentration

Figure 9 represents the effect of clay content on the viscosity variation rate. One can divide the graph into four unequal sections. The first section is in the range of shear rate less than $20\left(\frac{1}{s}\right)$ and indicates that sample $G$ is less viscous than sample $B$ but so small that it can be seen in the limited range of shear rate. The second section contains extensive range of tension rate and it can be considered as the most effective section of this graph for solution application to increase production of polymeric petroleum. This section identifies the shear rate from $20\left(\frac{1}{s}\right)$ to $100\left(\frac{1}{s}\right)$. As can see from the graph, the effect of clay addition in this section led to polymer concentration augmentation. The viscosity of two solutions approaches to each other as the shear rate increases which is similar to conformity in section third. Finally, when the shear rate increases again the sample B viscosity increases a bit more than the sample $G$.

To evaluate the effects of clay concentration increment on a low salinity system samples $\mathrm{K}, \mathrm{G}$ and $\mathrm{B}$ were compared (Figs. 10, 11). As it can be seen, in the first step of increasing clay concentration, viscosity increment is not sensitive (compare sample B with G). But in the case of increasing clay concentration in sample $\mathrm{K}$ two folded that of sample $\mathrm{G}$, the effects turn to positive and causes higher viscosity than that of sample $G$. we can conclude from the results that the clay supplying has negative effects on viscosity up to a certain rate and turns to positive effect over than that certain rate. The results for this section are 
Fig. 9 Solution viscosity vs. shear rate, to evaluate the effect of clay in low salinity system

Fig. 10 Solution viscosity vs. shear rate, to evaluate effect of increasing the clay content step by step in low salinity system
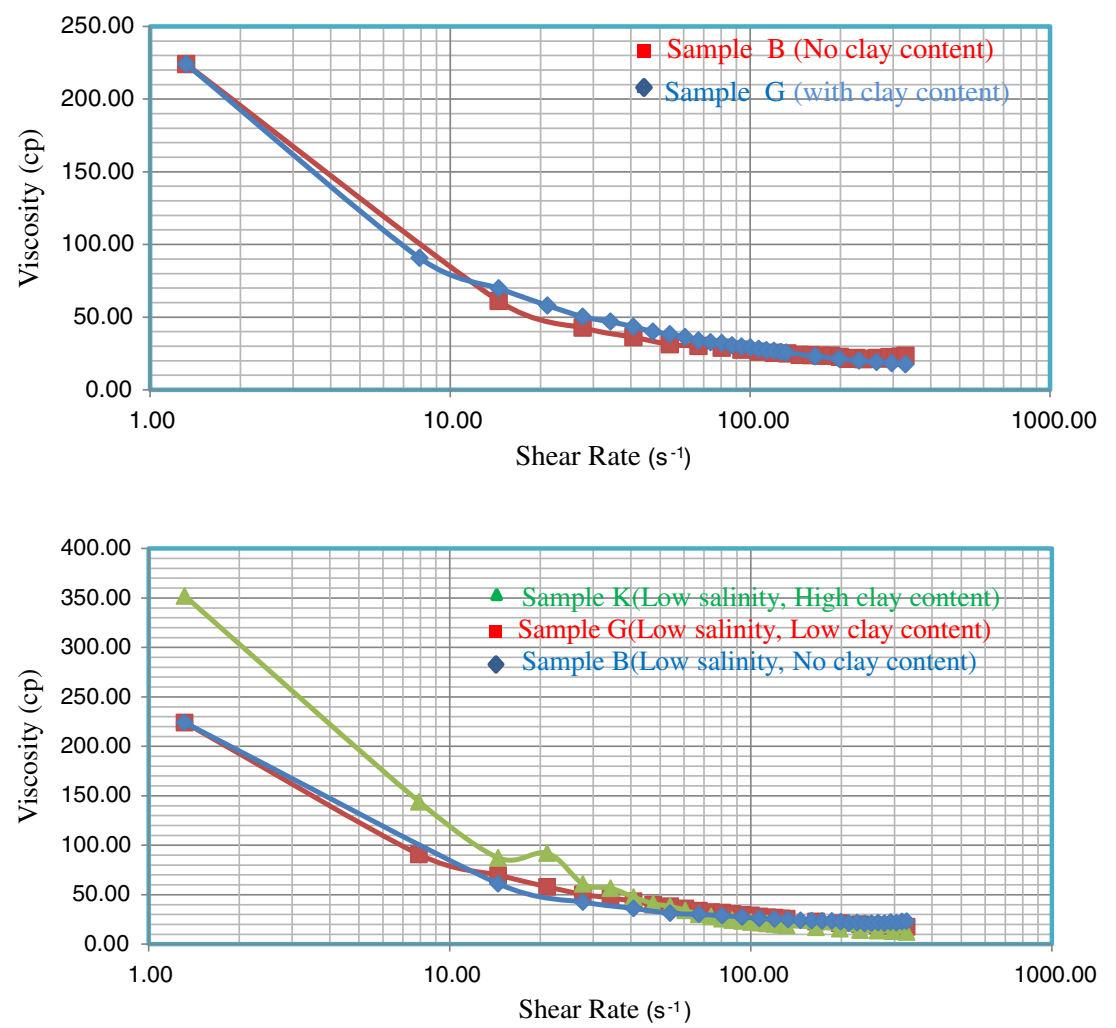
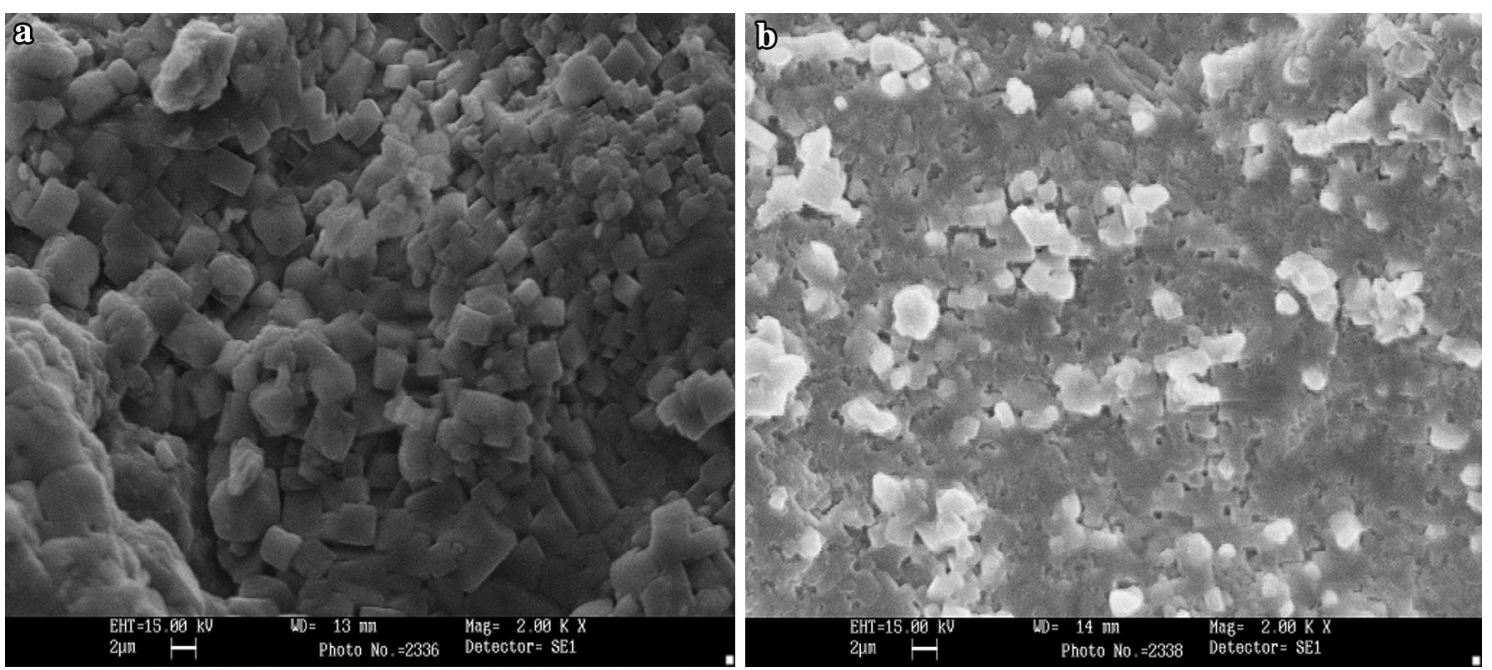

Fig. 11 SEM micrograms of the clay nanoparticles absorbed by the polymer solutions were prepared in 20,000 ppm brine; a Sample G, b Sample K

well aligned to that of previous one and show that this behavior is dominant in system both in low and high salinity conditions.

Also, three samples (F, H and D) provided to study gradual clay supplying effects on a high saline system. It is obvious from the graph that increase in clay concentration has negative effects on sample $\mathrm{H}$ so that in the case of $0.45 \mathrm{wt} \%$ increasing clay concentration, solution viscosity decreased. When clay concentration was two folded in sample F, clay supplement has positive effect and ends in viscosity increase comparing to the sample $\mathrm{H}$. We can conclude from the results that in high saline system, nanoclay can improve polymer viscosity (Figs. 12, 13). 
Fig. 12 Solution viscosity vs. shear rate, to evaluate the effect of increasing the clay content step by step in high salinity system

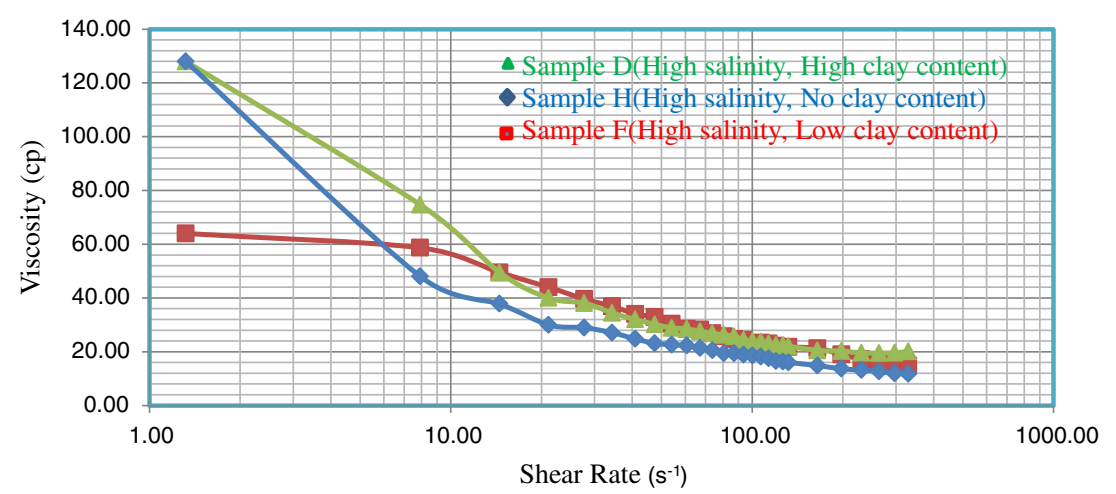

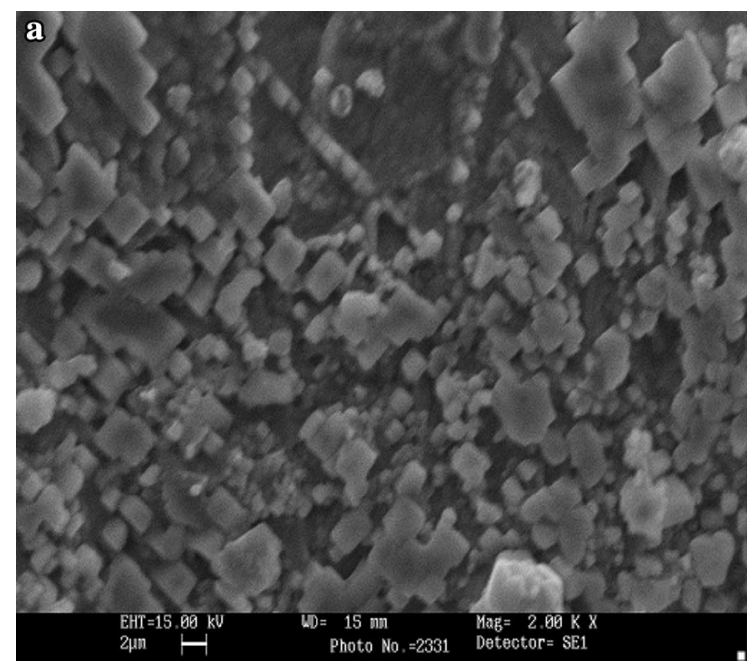

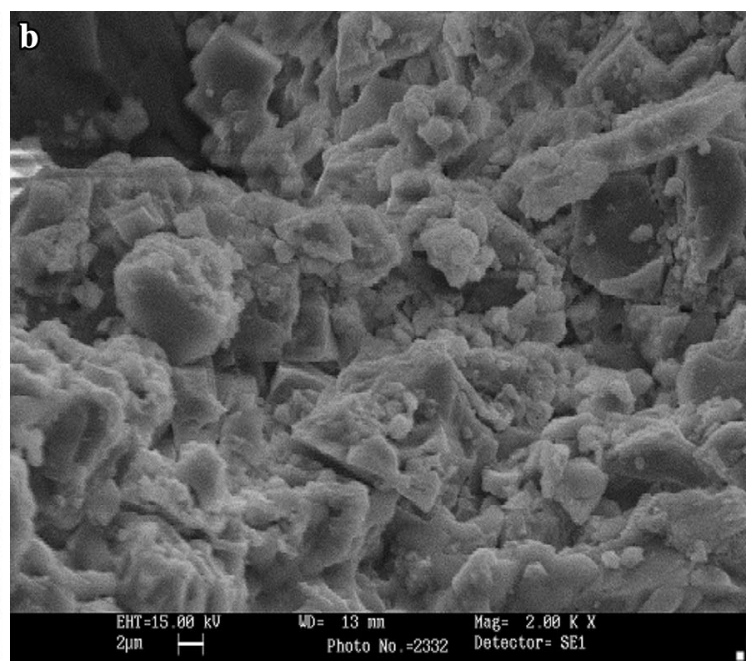

Fig. 13 SEM micrograms of the clay nanoparticles absorbed by the polymer solutions were prepared in 200,000 ppm brine; a Sample H, b Sample F

\section{Conclusions}

Based upon the conducted experiments, the following conclusions and observations are drawn:

- First, regarding the test results indicated that an increase in polymer concentration caused viscosity to increase.

- To study the effects of salinity on the polymer viscosity at the time of being ten folded its salinity, we found that in lower shear rates there is a negative effect from high salinity which became less by decreasing shear rate. The effect of clay supplying on a low saline system was that clay presence had negative effects on viscosity in an extensive range of intension rate and only in a limited range of shear rate it had positive effects.

- These results were investigated for a highly saline system which supported above-mentioned results and even a range of shear tension in which clay had positive effects in low salinity decreased in high levels of salinity.

- The salinity effects are also evaluated on a system with clay and constant polymer content that supported again negative effect of salinity increasing. An experiment was designed to evaluate this result so that clay concentration increase was highly intense and salinity went up, too. These changes were to achieve most optimal concentration for clay to retrieve the negative effects of the salinity. Results indicated that in certain range of clay concentration used in this test, the way of clay adding has positive effects on solution viscosity that should be increased to higher rates to be able to compensate all negative effects of salinity.

Acknowledgments Authors gratefully acknowledge Science and Research Branch of the Islamic Azad University, Khorasan Science and Technology Park and National Iranian South Oil Company for partial support of this work. 
Open Access This article is distributed under the terms of the Creative Commons Attribution License which permits any use, distribution, and reproduction in any medium, provided the original author(s) and the source are credited.

\section{References}

Al-Hashmi AR, Luckham PF, Heng JYY, Al-Maamari RS, Zaitoun A, Al-Sharji HH, Al-Wehaibi TK (2013) Adsorption of high molecular-weight EOR polymers on glass surfaces using AFM and QCM-D. Energy Fuels 27(5):2437-2444

Bazgir S (2006) Produced for use in polymer nanocomposites. Iran Rubber Mag 10:52

Cheraghian G, Hemmati M, Masihi M, Bazgir S (2013) An experimental investigation of the enhanced oil recovery and improved performance of drilling fluids using titanium dioxide and fumed silica nanoparticles. J Nanostruct Chem 3:78

Delshad M, Magbagbeola OA, Huh C, Pope GA, Tarahhom F (2008) Mechanistic interpretation and utilization of viscoelastic behavior of polymer soltions for improved polymer flood efficiency. In: SPE 113620 presented at the 16th SPE improved oil recovery symposium, Tulsa, OK, April 21-23
Ghannam M, Esmail N (2002) Flow behavior of enhanced oil recovery alcoflood polymers. J Appl Polym Sci 85:2896-2904

Green DW, Willhite GP (1998) Enhanced oil recovery. Society of Petroleum Engineers, Richardson

Khoeini M, Bazgir S, Tamizifar M, Nemati A, Arzani K (2009) Investigation of the modification process and morphology of organosilane modified nanoclay. Ceramics-Silikáty 53(4):254259

Magbagbeola OA (2008) Quantification of the viscoelastic behavior of high molecular weight polymers used for chemical enhanced oil recovery. MS Thesis, University Of Texas At Austin

Moradi H (2011) Experimental investigation of polymer flow through water- and oil-wet porous media. MS Thesis, University Of Stavanger

Pancharoen M (2009) Physical properties of associative polymer solutions. MS Thesis, University Of Standford

Rashidi M (2009) Physico-chemistry characterization of sulfonated polyacrylamide polymers for use in polymer flooding. Ph.D Dissertation at University Of Bergen

Satter A, Iqbal G, Buchwalter J (2008) Practical enhanced reservoir engineering. PennWell, Tulsa

Sun Y, Saleh, Bai B (2012) Measurement and impact factors of polymer rheology in porous media. Petroleum Engineering Program, Missouri University of Science and Technology, Rolla 